\title{
Relevance of Context-bound loci to Topical Potential in the Argumentation Stage
}

\section{EDDO RIGOTTI}

Faculty of Communication Sciences, University of Lugano

Via G., Buffi 13, Lugano CH 6900, Switzerland

E-mail:rigottie@lu.unisi.ch

\begin{abstract}
In relation to the task of making an expedient selection from the available loci within the topical potential that strategic manoeuvring envisages for the argumentation stage, this paper proposes a version of topics, which is both inspired to the traditional doctrine of topics and consistent with the theoretical framing and the methodological tenets that are proper of modern semantics and current theory of argumentation. The argumentative relevance of communication context in its institutionalised and interpersonal components is brought to light. Three main aspects are focussed on: the strong synergy of the topical and the endoxical components in argument construction, the use of topics in analysis and evaluation of arguments and the heuristic function of topics in the production process.
\end{abstract}

KEY WORDS: activity type, analysis, context, evaluation, heuristic function, production, strategic manoeuvring, taxonomy of loci, topics

\section{INTRODUCTORY REMARKS}

The topic of strategic manoeuvring, which is per se theoretically relevant, turns out to be particularly important for those who, like the author, develop their research in the context of communication sciences. The integration of the dialectical commitment with a rhetorical dimension corresponds to an extension of focus from a prevailingly analytical and evaluative concern to the acknowledgement of a specific feature characterizing argumentative production. Here, the commitment of reasonableness is combined with a party-bound attitude. In fact, if some roles in certain actualisations of the critical discussion demand a rather analytical and evaluative approach (this is basically the position of the decider in adjudication ${ }^{1}$ and of the other figures of "thirdness" like mediators and arbitrators), the role of the arguer himself always implies an attempt to prevail, rather than simply fulfilling a critical task. A neutral position is expected from someone beyond the parties and not from the parties themselves.

The focus on strategic manoeuvring is consistent with the pragmatic orientation of Pragma-dialectics: argumentation is not simply 
an inferential procedure; it is a (communicative) act embedded in a cultural-social (historical) context (van Eemeren and Grootendorst, 2004 , p. 52). Within this context, each arguer has his own aim and tries to realize it. Insofar as someone is an arguer, her aim is to bring her standpoint to victory in the respect and with the aid of a critical approach (van Eemeren and Houtlosser, 2002, p. 135).

The focus on strategic manoeuvring reconciles the dialectical and rhetorical approaches to the argumentative activity, which suffered, in particular in the last centuries, from a "sharp and infertile ideological division" (ibid., pp. 136-137). But it also fosters the professional implementation of Argumentation theory: the professional quality does not simply depend on the correctness of an argumentative strategy (within the tradition of Pragmatics we find in this relation the term felicity), but the success of such a strategy is pursued; by borrowing, again, another particular term from the tradition of Pragmatics, we could speak of happiness to point at a positive outcome of the argumentative intervention (Rigotti and Rocci, 2001, p. 50).

Rhetorical involvement is, by the way, precious in social life for various reasons. First of all, the desire of winning is not necessarily originated in the mind of an intentional and perverse manipulator: it can be rather the sign of a sincere commitment to a certain position, which entails the willingness to "prove" its validity. Moreover, the dignity of the rhetorical involvement is also implied by the fact that the right to a defender is ensured in all advanced legal systems to each defendant. This right, which is acknowledged even to manifestly guilty parties, is explained by the fact that their very reasons are helped to emerge only if some arguer applies her rhetorical effort to make them prevail. The same fact justifies that sort of "faith" in the effectiveness of the dialogic approach to the resolution of differences of opinion, which underlies the pragma-dialectical interpretation of reasonableness. ${ }^{2}$

\section{STRATEGIC MANOEUVRING AND THE ARGUMENTUM-VERSION OF TOPICS}

I will now point out those aspects of strategic manoeuvring in relation to which the Argumentum ${ }^{3}$ group is developing its contribution.

The role of strategic manoeuvring can be understood only if considering the specific opportunities offered to arguers in each stage of critical discussion for simultaneously saving the dialectical aim of the stage and their party-bound interests (van Eemeren and Houtlosser, 2005a). In each stage, strategic manoeuvring develops along three coordinated but distinct dimensions: "making an expedient choice from the options constituting the topical potential", i.e. from "the set of relevant alternative moves available to a party in that stage of the resolution 
process"; "selecting a responsive adaptation to audience demand"; and "exploiting the appropriate presentational devices" (ibid., p. 29). Our recent research within Argumentum at the University of Lugano has concentrated on a particular phase of strategic manoeuvring, which concerns topical potential within the argumentation stage. This phase envisages the choice of a specific line of defence of one's standpoint "that involves a selection from the available loci that best suits the speaker or writer" (ibid., p. 30). The tradition of argumentation studies has devoted significant attention to this task through the elaboration of a doctrine of topics (see Aristotelis, 1949, 1958, 1959; Hispani, 1947; Hubbel, 1949; Quintiliani, 1970; Stump, 2004). This doctrine is, in fact, ultimately aimed at constructing an "argument generator" that should provide the arguer with a "euporia" (richness) of arguments supporting his standpoint.

Within Argumentum, we propose (Rigotti and Greco, 2006) a model of topics that, though inspired by the ancient and medieval tradition, taking into account the developments of modern semantics and pragmatics, is strictly connected with the theoretical and methodological debate characterizing the contemporary theory of argumentation. ${ }^{4}$

The system of topics included in Argumentum represents a sort of engine of this production-oriented model, as it simulates the process of generation of relevant arguments. A diagram, which an indeed vague likeness induced us to name "Fishbone", and which plays a relevant role in orienting users' navigation, represents the constitutive components of the model in the framework of their relationships. As the Fishbone model privileges the point of view of production of a single argumentative intervention, it needs to be correlated to the general framework of critical discussion. (Figure 1)

In order to situate topics within Argumentum, I will briefly illustrate the main components of the "fishbone" model.

The first rectangle is mainly devoted to the communicative context of an argumentative intervention; context, in fact, dictates conditions and aims of the argumentative intervention, and all its relevant factors (from the subjects involved, to the interaction field - social context of interaction -, and to the communicative practice) must be taken into account in order to design a rhetorically and dialectically adequate argumentative intervention.

As the notion of communication context is both hugely relevant and considerably complex, Rocci and I have devoted to it an analysis that has been expounded in a specific paper (Rigotti and Rocci, 2007). Here, it might be useful to briefly outline the results of such an analysis, starting with a scheme representing the articulation of this notion: (Figure 2) 


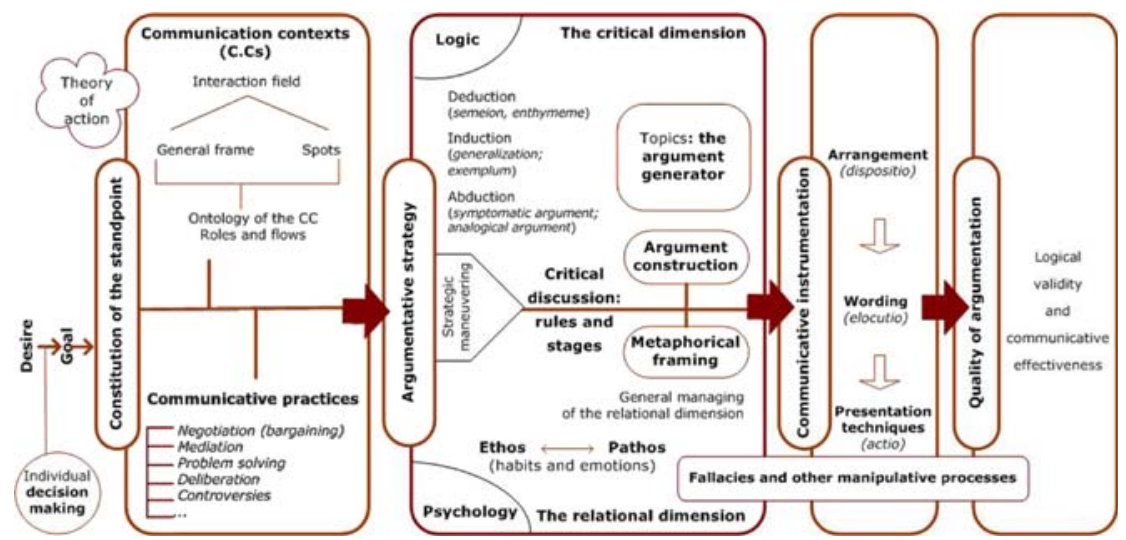

Figure 1. The "fishbone" model of argumentative intervention.

Communication context is constituted by two relevant dimensions, which can be characterized as institutionalised and interpersonal respectively.

1. The institutionalised dimension has been focused on within the pragmadialectical approach (van Eemeren and Houtlosser, 2005b) through the notion of activity type, introduced by Levinson (1978). The complex notion of activity type includes in turn two components that, in our opinion, deserve to be kept distinct.

1.1. The first component is represented by the interaction field, i.e. by that piece of social reality (in Searle's (1996) terms) where the argumentative interaction takes place. The interaction field is defined by specific (hierarchically organized) shared goals, which all the interagents share

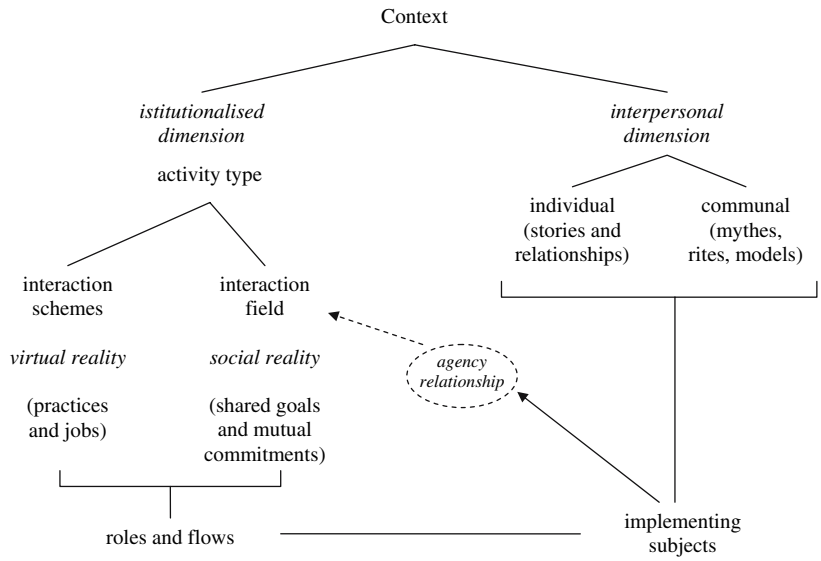

Figure 2. The communication context. 
beyond their individual goals, and which define the interagents' mutual commitments. For instance, a business is distinguished from other kinds of institution (hospitals, universities...), because its main shared goal is making a profit.

1.2. The shared goal which is pursued by the interaction field is de iure the final aim of all the institutional interactions occurring in the interaction field itself. The interaction field operates through a series of interaction schemes which aim at the fulfilment of the shared goals. Interaction schemes presuppose and entail corresponding communicative practices or, properly, communicative interaction schemes (like deliberation, negotiation, consulting, problem-solving, adjudication, mediation...), which, at least in some cases, also constitute proper jobs. For instance, a business needs to make strategic decisions about its financial activities in order to reach its main goal of making profit; often, the process of decision-making is made through the deliberation by a group of people. But the communicative practice of deliberation is also applied in other interaction fields (such as a city council, a university department, etc.). The communicative practice is constituted as an interaction scheme aiming at fulfilling the shared goals (the main one and the subordinate ones) defined by the interaction field; the practice involves rules of interaction and specific communicative and non-communicative tools that have been established in the history of the practice itself.

The implementation of interaction schemes within interaction fields generates a network of roles that are linked to each other through correspondent communicative flows.

2. The roles that are thus generated are "embodied" by implementing subjects that can be individual or collective. In relation to implementing subjects, the relevance of another component of context arises: the interpersonal dimension. This second component has actually to do with the human factor of context. An implementing subject is not to be understood as a simple "filler" of the institutional role, endowed with the required competences: indeed, for each real (individual or collective) implementing subject, the subjective dimension always exceeds the institutional role. The subject maintains his or her own interests and goals, which may be congruous with the role itself, or may be conflicting with it. It is, indeed, a typical case of agency relationship. ${ }^{5}$

In the interpersonal dimension two types of interpersonal solidarity take place. The first one concerns the relationships between individuals: living and working together within the same interaction field originates various types of stories, in which experiences are shared and relationships established; such stories may also influence the institutional dimension. The second type of solidarity concerns the particular 
link of individuals "belonging" to the community, which creates myths, rites and models (Cantoni and Di Blas, 2006, pp. 233-237); in other words, the proper culture of the interaction field.

The relevance of communication context to the argumentation strategy is justified by several reasons:

- Within context the issue emerges in relation to which the difference of opinion generating the standpoints arises;

- Context is the primary source for defining the strategies in the opening stage;

- Context provides arguers with that explicit or implicit information protocol from which many proper endoxa are drawn.

The second rectangle of the fishbone model is explicitly inspired by strategic manoeuvring, as it is fully devoted to the design of argumentative strategies. Two strictly complementary dimensions are considered: the critical and the relational ones. Each of them offers the possibility of an interdisciplinary enrichment. The critical dimension naturally refers to logic, ${ }^{6}$ which is expected to facilitate the assessment of the inferential validity of argumentative procedures. The relational dimension, moving from the Aristotelian notions of pathos and ethos, largely corresponds to the second level of strategic manoeuvring (adaptation to audience demand). In particular, it could be profitably connected with those investigations in social psychology that are devoted to persuasive effectiveness of argumentative strategies (Petty et al., 1983; Petty and Cacioppo, 1986). The main component of this rectangle clearly coincides with topics, which offers a tool for managing the topical potential, i.e. the set of relevant alternative moves within the argumentation stage. The model of topics worked out in Argumentum will be focused in the following of this paper.

The third rectangle concerns the communicative instrumentation that "dresses up" the argumentative strategy. This dimension is strictly connected to the presentational devices within strategic manoeuvring and articulates this dimension into the arrangement of the arguments in a rhetorically effective order (dispositio), stylistic choices at the linguistic level (wording or elocutio), and non-verbal communication, including body-language (actio), and other presentational techniques (like graphics, possible audio-visual supports, and so on).

Finally, the fourth rectangle intends to represent the auto-critical reflection which needs to be developed after performing the argumentative intervention within an actual argumentative discussion, in order to improve one's argumentative practice by learning from experience.

The problem of fallacious argumentation strategies of course affects all of the three last rectangles. 
I will now consider topics, more specifically, being the kernel of the Fishbone model, and particularly relevant to the point of view of strategic manoeuvring in the argumentation stage. Indeed the system of topics plays a crucial role with regard to the three main functions of the model:

- Analysing argumentative interactions by identifying the inferential processes they activate;

- Evaluating the dialectical and rhetorical effectiveness of argumentative discourses;

- Supporting the planning and construction of argumentative interventions by offering a rich toolkit of alternative argumentative instruments. By the way, this last function significantly corresponds, in the argumentation stage, to the endeavour of strategic manoeuvring.

This component of Argumentum consists of two main parts, each one of which is in turn subdivided into several sections.

The former part is devoted to the theoretical framework and the analytical instruments necessary to face topics. Firstly, a definition of some key notions of topics is proposed and discussed. Secondly, a relevant section is devoted to the use of various semantic instruments for establishing the ontology of the standpoint in its syntagmatic and paradigmatic dimensions and its modal status (see the borderline between what is already ascertained and what is problematic, Rigotti and Greco, 2006). Finally, the inferential structure of a locus is analyzed by focusing on the maxims arising from different knots of this ontology (hooking points) and on the endoxa they evoke.

The latter part outlines the argument generator, which includes a taxonomy of loci, and the presentation of one or more maxims arising from each locus, with their application to specific arguments by mapping maxims onto the information protocol offered by the context. (Figure 3)

The model has been more systematically illustrated in other occasions (Rigotti and Greco, 2006; Rigotti, 2007). Here, I will limit myself to the presentation of its fundamental traits; in particular I shall focus on the relationship between the argument choice and the communicative context within which the argumentative intervention takes place.

\section{DEFINITION OF SOME KEY NOTIONS}

I will start defining the key notions our model of topics is based on: topics, locus, endoxon, hooking point, maxim, argument.

Traditionally, topics indicates a systematic method of finding arguments. Aristotle identifies it with "a method according to which we are 


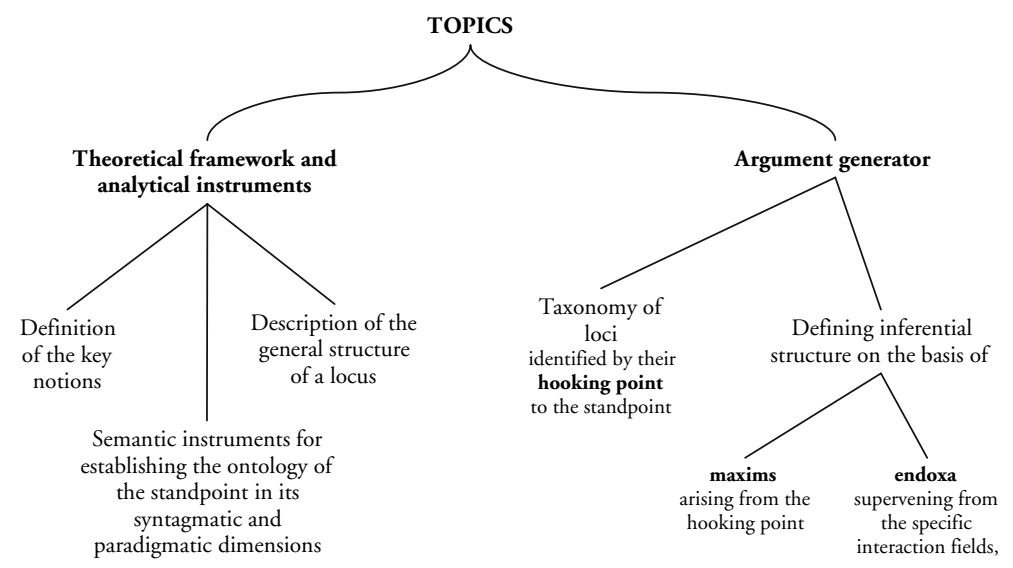

Figure 3. The main components of topics.

able to put forward arguments about any standpoint (problema) starting from propositions which have already been accepted (ex endoxon)" (Topics, 100a 1 see Aristotelis, 1958). Now, as topics and, more in general, rhetoric were considered in antiquity as "technai", i.e. as arts and not as sciences, their theoretical purpose was not put in the foreground. In order to emphasise the scientific nature of topics, as required by its connection with argumentation theory, we focus on its theoretical commitment by adopting the following definition: Topics is the component of argumentation theory by which ideally all ${ }^{7}$ (theoretically possible) relevant arguments in favour and against any standpoint are generated by specifying their inferential structure through a system of loci (Rigotti and Greco, 2006).

Two fundamental notions are involved by this definition: standpoint and locus.

A standpoint is a statement (simple or complex) for whose acceptance by the addressee the arguer intends to argue.

Two remarks are necessary.

Firstly, a standpoint is a particular type of statement which (1) did not yet receive a shared justification (neither by evidence nor by a previous inference); (2) is bound to a commitment of immediate justification by an inferential procedure (it is candidate to figure as a conclusion).

Secondly, a standpoint is always a statement, even though it can be subject to different modalities and thus provide logical equivalencies to other types of utterance (pieces of advice, orders, questions, and so on).

Being the basic constituent of our model, the notion of locus plays a fundamental role. A locus ${ }^{8}$ is a "sub-generator" of argumentative procedures consisting of one or more maxims in the form of truth 
conditions that bind the truth value of the standpoint to the acceptance by the considered public of propositions referring to specified aspects of the ontology of the standpoint.

It is worth noticing that the inferential process cannot be activated if the maxim is not combined (crossed) with propositions that have already been accepted by the considered public, as they typically correspond to opinions that are considered within the community. This component of the argumentative procedure was named by Aristotle endoxon: "what is already within the shared opinion". It is interesting to directly quote, in this relation, the definition given by Aristotle (Topics, 100b. 21, see Aristotelis 1958): endoxa are opinions that are accepted by everyone or by the majority, or by the wise men (all of them or the majority, or by the most notable and illustrious of them)". A modern translation could be: "an endoxon is an opinion that is accepted by the relevant public or by the opinion leaders of the relevant public".

In the above-proposed definition of locus, the notions of hooking point and maxim become relevant:

The aspect of the standpoint that the maxims of a certain locus refer to represents the hooking point of the locus to the standpoint and gives the name to the locus itself.

Maxims are implications establishing a connection between the truth value of a hooking point and a standpoint of the form $\mathrm{p} \rightarrow \mathrm{q}$, that generate inferential processes; each inferential process defines, within the locus, the form of a subclass of arguments that are produced in connexion with proper endoxa. All the maxims of the same locus share the same hooking point to the standpoint.

Finally, we arrive at the definition of argument: we consider an argument as the actual application of a maxim to one or more proper endoxa, thus deducing the standpoint from the maxim for a certain public that shares the above-mentioned endoxa.

Let us consider an example that could help us recognize the relations between these last key notions of topics. If we consider the locus from the final cause, we observe that all the arguments it generates origin from a particular aspect of the standpoint: the final aim of the action referred to by the standpoint. But, depending on the presence or absence of a finality (motive), it is, first of all, possible to state whether the situation the standpoint refers to is an actual action or rather an event, e.g. an involuntary behaviour ("You just stepped on my foot!" "I didn't do it on purpose!'). However the same locus can generate other kinds of arguments. Let us suppose that the nature of an action has already been defined and that the arguer's aim is to determine whether this action can be described as murder or as a case of self-defence. The argumentative process will develop in the 
following way: if it has been ascertained from a number of circumstances that the victim was evidently not capable of causing serious damages to anybody, then the locus from the final cause allows concluding that the hypothesis of murder is true. Both procedures, as different as they may be, are established in relation to the same hooking point: the finality of the action. It is clear that within the same locus, i.e. by referring to the same moment of the semantic-pragmatic structure (ontology) of the standpoint, different kinds of argument can be found. Their variety depends on two mutually connected factors:

(1) the border which is posed in the standpoint between what is already ascertained and what is still disputed (in the first example, the status of action has not yet been ascertained and is thus being disputed, whereas in the second example it is taken for granted and what is disputed is the quality of the action);

(2) the specific maxim. For example, within the locus of the final cause, referring to a different border between ascertained and disputed, we have found two different maxims: (1) If a behaviour does not have a final cause, it cannot be defined as action in a strict sense; (2) If for an action, the final cause that is pretended is evidently meaningless or incompatible with the actual circumstances, another final cause must be identified.

\section{TAXONOMY OF LOCI}

The taxonomy of loci proposed in Argumentum is not illustrated in detail here; I will limit myself to outline it concisely and mention other presentations (Rigotti, 2007; Rigotti and Greco, 2006)

In the Medieval literature on topics, loci were distinguished, according to their proximity to the standpoint, into intrinsic, extrinsic and middle loci.

In general, the basic distinction between intrinsic and extrinsic loci can be found in Cicero: "alii in eo ipso de quo agitur haerent, alii assumuntur extrinsecus" (M.T.Ciceronis, Topica, see Hubbel, 1949) Our taxonomy is however closer to the typology formulated by Themistius and followed by Boethius (De topicis differentiis, see Stump, 2004): (1) the loci taken from those factors that are directly established by the standpoint (vel ex ipsis sumantur quae in quaestione sunt constituta), (2) those loci that are taken from the outside (vel extrinsecus ducantur), and (3) those loci which can be found almost on the borderline between the previous two (vel quasi in confinio horum posita vestigentur).

Moreover, the intrinsic loci include not only those things which are referred to in the standpoint, but also those that condition the state of affairs denoted by the standpoint, and that either follow it or come together with it. They correspond to all those aspects that constitute 
the possible fragment of the world expressed in the standpoint or that coexist with it. Exploiting the notion of syntagm, introduced in modern linguistics to mean the set of relations in praesentia, we speak of syntagmatic loci to indicate all the classes of arguments that refer to aspects that are ontologically linked to the standpoint, either directly or indirectly, such as the extensional relations of terms, dependent on the semantic content, on the hierarchical taxonomy of predicates, on the relationship between the whole and its constituent parts; included in this group of loci are also the classes of arguments which assume as their hooking point those pieces of world, traditionally called causes, effects, circumstances and concomitances, that condition the state of affairs the standpoint refers to.

In Themistius' and Boethius' (ibid.) tradition, the description of the extrinsic loci, on the other hand, is undoubtedly more vague: "Extrinsic loci are not so "separate" and detached as to prevent the vision, from a certain perspective, of what constitutes the situation concerned by the standpoint" (Non sunt ita separata atque disiuncta, ut non aliquo modo quasi e regione quadam ea quae quaerentur aspiciantur). When considering the loci included within the extrinsic ones (similarity, opposition, major-minor, termination and setting up), it is nonetheless quite clear that they correspond to those relations in absentia (of alternativeness) defined by modern linguistics as paradigmatic. Thus we speak of paradigmatic loci referring to classes formed by arguments that are based on paradigmatic relations, both of opposition (see the notion of semantic paradigm in Rigotti and Greco, 2006) and of analogy (similarity).

As regards the loci medii (also indicated as mixti), they are characterized by being on the borderline (in confinio) between extrinsic and intrinsic ones. The name complex loci seemed to be more adequate to account both for the frequent contamination they show between paradigmatic and syntagmatic loci, and for the frequent inclusion of extradiscursive elements. A prime example of this should be considered the locus from authority, which, pointing to the moral and $\backslash$ or cognitive quality of the "producer" of the message, refers first of all to the syntagmatic locus from agent as a subtype of the locus from efficient cause; nevertheless, the aspect taken into consideration does not refer to the content of the standpoint but to the communicative situation in which the standpoint is being discussed. Among the complex loci can be considered also the " $a d$ " arguments, having in general a dialectically deviant nature. Derivates and conjugates are more rhetorical than dialectical: derivates refer to the so called etymological figure ("If he is an entrepreneur, he should stick to managing enterprises and not pretend...") and derive indeed their argumentative power from semantic implications of different nature and different inferential strength (as in 
our case, it is frequently a matter of the relation between the status of a certain being and its tasks); ${ }^{9}$ conjugates instead draw on the semantic relations implied by morphological patterns ("Those who fought against us have lost, then those who will fight against us will loose") and activate one or more syntagmatic or paradigmatic loci.

We propose the following diagram to represent the taxonomy of loci, where the three domains of syntagmatic, paradigmatic and complex loci appear with their articulations: (Figure 4)

\section{TOPICS AS IMPLEMENTATION OF THE TOPICAL POTENTIAL IN THE ARGUMENTATION STAGE: AN ANALYTICAL APPROACH}

Within this paper, as said, I concentrate on the topical potential in the argumentation stage. In the adopted definition, an argument is an

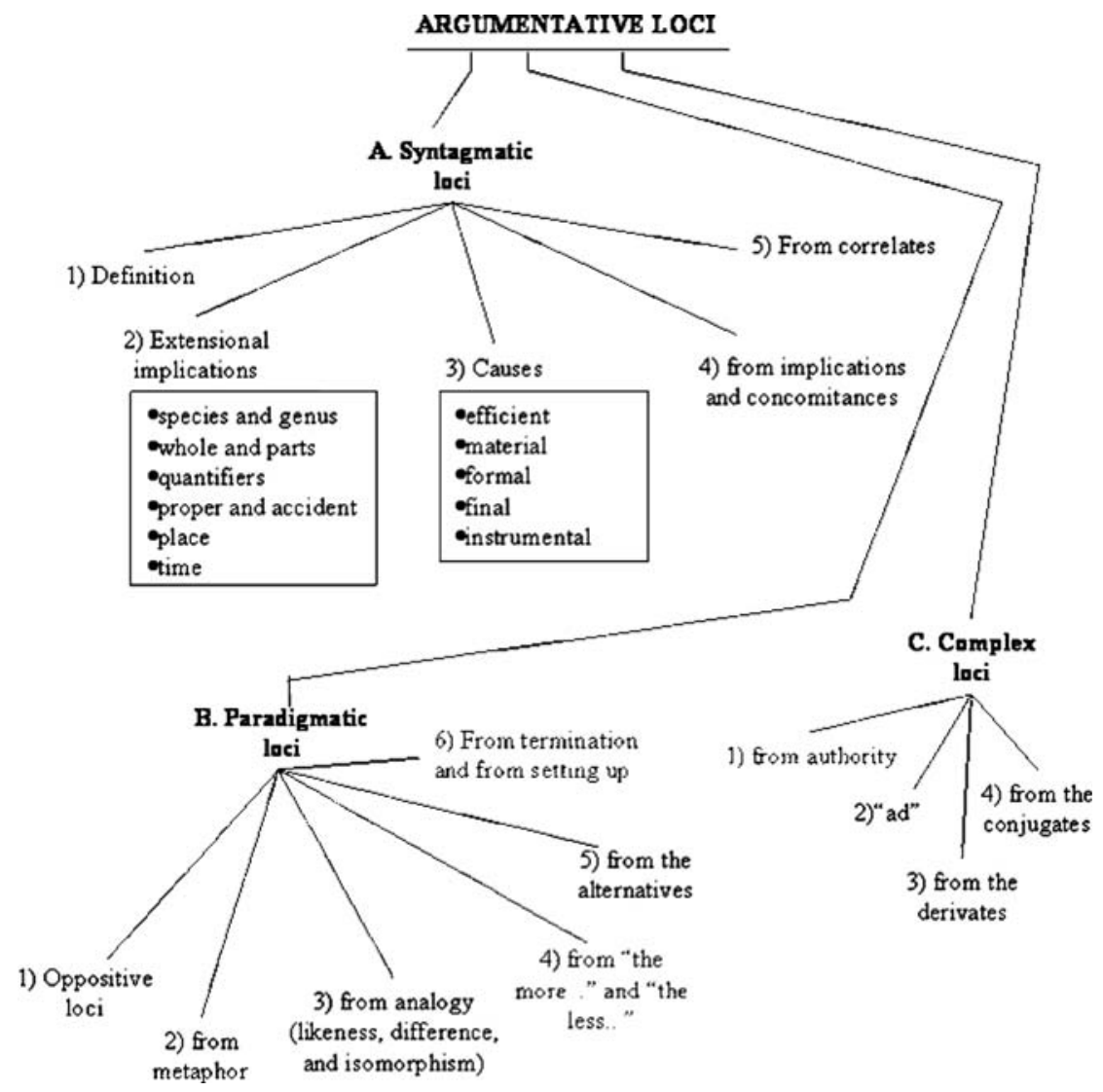

Figure 4. Taxonomy of loci. 
actual application of a maxim to one or more proper endoxa, inferring the standpoint from the maxim for a certain public that shares such endoxa; my hypothesis is that, in relation to the endoxical component, the communication context of the argumentative interaction represents a rich source of loci. In the following, I will bring some examples where this relevance clearly emerges.

\subsection{A Happy Experience of Strategic Manoeuvring}

In the first example, the only one I shall analyse in detail here, the context is represented by a particular type of institution, and the practice is a quasi-negotiation for settling an initial institutional conflict. Presentational techniques are, by the way, significant and a successful case of strategic manoeuvring is exemplified. For me personally, it is also the occasion to remember a dear friend, the former director of the Department of Education and Culture of Canton Ticino, Giuseppe Buffi. ${ }^{10}$

The argument concerned is based on a paradigmatic locus; this locus belongs to the general class of analogies, together with the loci from likeness and from difference: the locus from isomorphism. This argument is drawn from the contemporary history of Italian Switzerland: in 1996, when the University of Lugano was founded and Giuseppe Buffi presented the unexpected novelty to the representatives of the Cantons with an older university tradition, in fact he exploited the locus from isomorphism to back an ex post facto announcement: "It is true: the Canton of Ticino never discussed its project of building a university with the Confederates, but it decided and planned its realization and only then they made the announcement. However, exactly the same happens when a young couple wants to have a child: they conceive and then run to announce it to their parents".

An adult son and an adult daughter, when building a new family, though still strongly bound to their family of origin, are themselves responsible for their own choices. So Ticino, adult son of the Confederation, consciously deciding, gives birth to a new university and only then makes the announcement to the "parent-cantons".

In this case, the simile rests on an accepted isomorphism of the two systems. Two conditions are entailed by an isomorphism: (1) there is a bijective mapping between the two systems (there is one and only one element of the second system corresponding to each element of the first one) and (2) if in the first system a relation $\mathrm{R}$ holds between the two elements, in the second one an analogous relation $\mathrm{R}^{\prime}$ holds between the correspondent elements.

In the considered example, the argumentation refers to an accepted isomorphism between the enlarged family and the Swiss Confederation. On the one hand, in this isomorphism the parents (the families of 
origin) correspond to the older cantons - cantons having a consolidate university tradition - while the Ticinese community corresponds to the young couple; on the other hand, the responsibility-bound autonomy of the young couple towards their parents is analogous to the responsibility-bound autonomy of Ticino towards the older cantons. The maxim activated by this argument establishes in general that, given such an isomorphism, if, $\mathrm{P}$ holds for two members of one system, $\mathrm{P}$ ' is expected to hold for the correspondent members of the other system. Thus, if a certain behaviour is legitimate among certain members of the enlarged family, an analogous behaviour is legitimate, in analogous circumstances, also for the correspondent members of the Swiss Confederation: as the young couple does not usually communicate the plan of conceiving a baby to the parents, but the announcement is made ex post facto, so Ticino is expected to communicate the foundation of a new university to the older cantons only after having already given life to the new institution. The following synergic representation highlights the different components and the steps of the argument supporting Buffi's unexpressed standpoint (it has been natural for the youngest canton not to have announced the plan of opening a university before the decision had been made): (Figure 5)

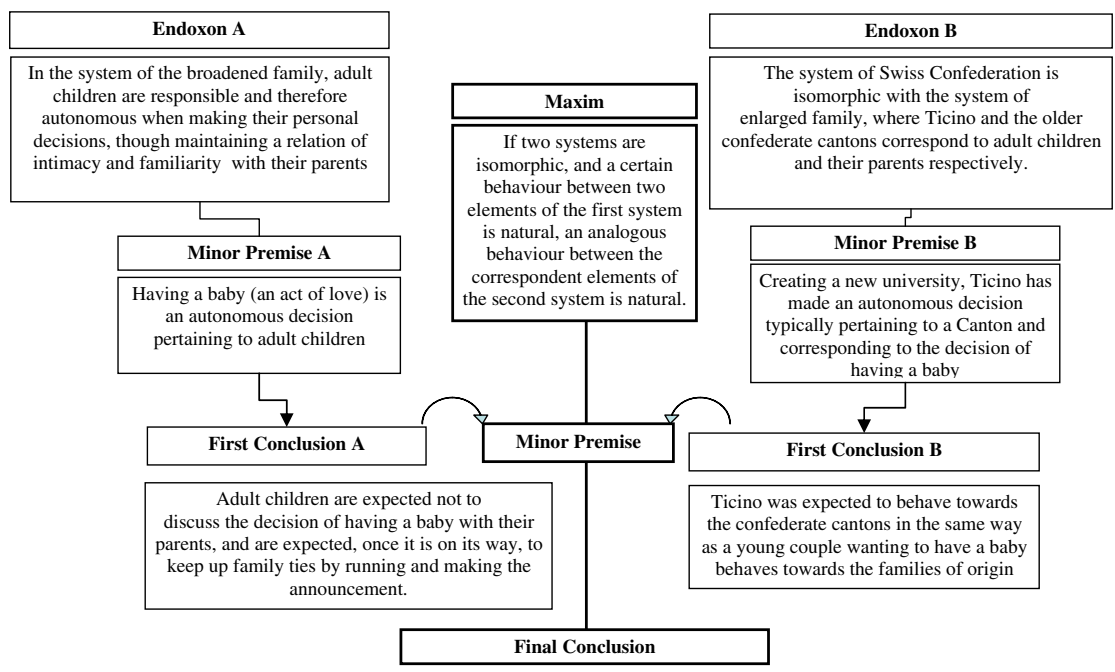

Ticino's behaviour - deciding autonomously on the creation of it university, and then announcing it promptly to the confederate cantons - is natural

Figure 5. Representation of of Buffi's argument showing the synergy of the topical and endoxical components. Minor premises are connected to the endoxa working as their major premises through a conjunction relation. The same relation connects the first conclusions $\mathrm{A}$ and $\mathrm{B}$. 
Our analysis turns out to show that the inferential process at the basis of this argumentation is quite complex. The inferential structure of the argument consists of three different inferential procedures, one of which assumes a maxim of the locus from isomorphism as its major premise, whereas the other two assume as many endoxa as their major premises. Let us start considering the syllogism based on the maxim: in order to reach the desired conclusion, it is necessary to assume a complex minor premise, corresponding to the conclusions of two endoxa considering the system of a family, on the one side, and the family-like structure of the Swiss Confederation, on the other side:

1. Adult children are not expected to discuss the decision of having a baby with their parents, and are expected, once it is on its way, to keep up family ties by running and making the announcement.

2. Within the isomorphic system of Switzerland, Ticino was in an analogous position towards the older Cantons.

These statements need in turn to receive a justification, which can be obtained respectively from two other inferences that are intertwined and combined with the inferential process of the maxim (originated by the locus). Such inferences are constituted by syllogistic structures with endoxa (endoxon A and B respectively in the diagram), in the place of the major premises. In the place of the minor premises, there are more specific propositions (minor premises A and B in the diagram), linking the subjects of the endoxa ("adult children" and "Ticino"respectively) to the terminus medius" (in A "responsible and autonomous in decisions of one's competence"; in B: "making a decision of creating a university is autonomous analogously to the decision of making a baby"), and thus deriving the First Conclusions A and B. The first conclusions "prove" that a certain aspect of the standpoint (Ticino's behaviour towards its confederates) has a certain property (being analogous to adult children behaviour towards parents in relation to the decision of having a baby), which is exactly what the maxim "lacks" in order to generate the final conclusion. The small curved arrows in the diagram represent the fact that the final conclusions are exploited together as minor premise associated to the maxim for generating the final conclusion.

Such an argument does not base its inferential strength simply on the locus from isomorphism, but in the degree of reliability of the isomorphism between a family and the Swiss Confederation (endoxon B), and in the degree of reliability of the endoxon concerning the behaviour of a young couple (endoxon A). Whereas the endoxon A refers to a generally shared connection between responsibility and autonomy of decisions, and shows a certain strength, the endoxon B is strictly connected to the institutionalised structure of the interaction field, 


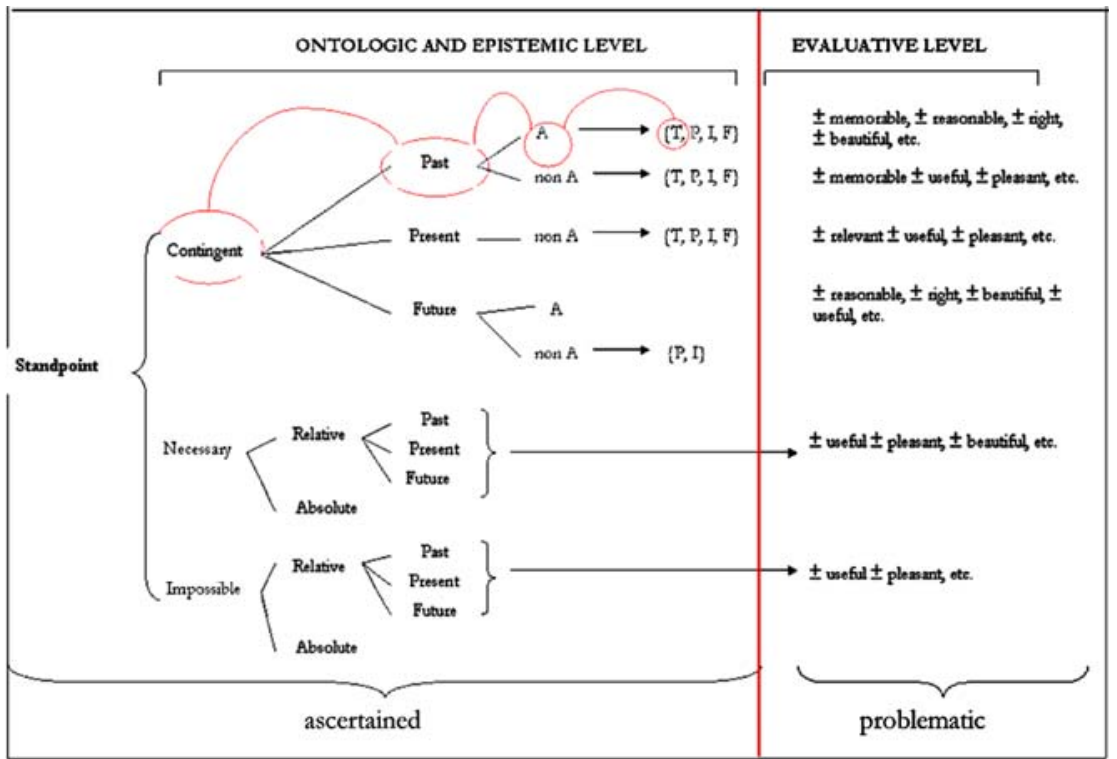

Figure 6. Defining the modal status of the standpoint.

which is constituted, in this case, by the Swiss Confederation. Notice that the minor premise B refers both to a juridical feature of the specific interaction field concerned and to its cultural identity, and does not come from the inferential scheme of the locus, as it is a datum emerging from the actual context of interaction.

The awareness of the Swiss cantons of forming a family-like community is clearly evoked: in relation to this component, Buffi's argument may at least partially sound as a warning ( $O f$ course, none of you would doubt that we are a family!). And this argumentation can easily develop into other possible moves: the newborn university is, in this case, the little grandchild of the elder Swiss universities and must therefore be recognized and loved.

Such an example presents the application of a locus which is strictly connected both to the institutionalised and to the interpersonal dimensions of the Swiss nation. On the one hand, Swiss federalism is evoked with its juridical implications (each canton, in fact, has formally the right to open a university); on the other hand, the feeling of willingly belonging to the Swiss community is activated through the metaphor of the enlarged family. We could also notice that the institutional aspect was per se legally sufficient to justify Ticino's behaviour, but the choice made by Buffi within the topical potential allowed him to save and consolidate a cordial relationship with the other cantons. 


\subsection{Other Context-Bound Applications of Loci}

Generally speaking, the activation of loci in the construction of an actual argumentative strategy may either involve both dimensions of context at the same time, or, alternatively, select endoxa that are more directly related to the one or to the other dimension. In other words, a single argument based on a locus might specifically evoke the interpersonal dimension only or the institutional dimension only.

If we take as an example the locus of termination and setting up, which is largely employed in conflict resolution practices as mediation, it emerges that this locus is often applied by mediators both to the institutionalised and to the interpersonal commitments between the conflicting parties (see Greco Morasso, forthcoming). A very frequently employed maxim of this locus can be formulated as follows: "If a certain relationship is precious, it should not be interrupted". Mediators may hook this maxim to the parties' interpersonal relationship, if it has been acknowledged to be positive (friendship, love...) or they might evoke an institutionalised endoxon, like the parties' involvement in an agency relationship which is working well, or in a business which makes a good profit, and so on. Recent research on mediation (Greco Morasso, 2007) has identified the application of this locus to both dimensions in mediation sessions. Consider the following two passages, which refer respectively to the interpersonal and to the institutionalised dimensions:

M: [But let me] ask a question here. You know what the value of your friendship is (.) but ah:: (..) if (..) things didn't work out (..) today (.) will that friendship be lost?

M: ... And all the time, I think, keeping in mind that one of the things you really want to do is - you've got a golden goose here. And it would be crazy to kill the golden goose. P1: That's what I've tried to tell him.

\subsection{The Generator as a Discovery Instrument: Towards a Production- oriented Approach}

So far, our generator of loci has presented itself as a theoretical tool for the analysis and critical evaluation of argumentative texts.

Indeed, topics has been aiming since its origin also at another significant purpose: finding out the relevant arguments for supporting a certain standpoint. Such a purpose has regained its relevance in contemporary theory of argumentation: a heuristic effort devoted to find out those arguments that can support one's standpoint is presupposed by the notion of strategic maneuvering as defined by van Eemeren and Houtlosser. 
Let us see how we could justify a simple standpoint, which could appear in an advertising campaign: "This butter is natural". It is possible to say that our standpoint is an assertive proposition, which states the existence in reality of a certain state of affairs. This proposition is constituted by a predicate with one argument place, which is occupied by a nominal structure:

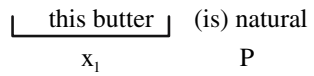

Semantic analysis allows defining the value of natural as a one-place predicate, presupposing a product of human activity (in particular, food). ${ }^{12}$ This is actually the case in our standpoint, where natural is said of "this butter".

Applying the diagram represented in figure 6, we get to establish that the borderline between what is ascertained and what is to be discussed in the standpoint lies at an evaluative level: in fact, the question is whether the butter is natural or not, while it is ascertained at the ontological and epistemic levels that butter is a product of the human activity. Clearly if there is a product to advertise, this means that the action of producing it has actually been performed in the past.

Argumentation has the nature of a discourse that rests on something which has already been ascertained in order to define the truth value of a certain standpoint. Thus, for the evaluation of a standpoint it is necessary to go back to what is certain, i.e. to the unquestionable information available. If there were no shared information with respect to the standpoint, the justification of the standpoint would be impossible.

However, the latter case seldom occurs. Usually a certain information protocol is available, which seems to be referred to the standpoint in a more or less direct way. If we consider the standpoint in our example, which can be classified as an advertisement, we can imagine that the team responsible for creating an advertising campaign had been given the following information protocol:

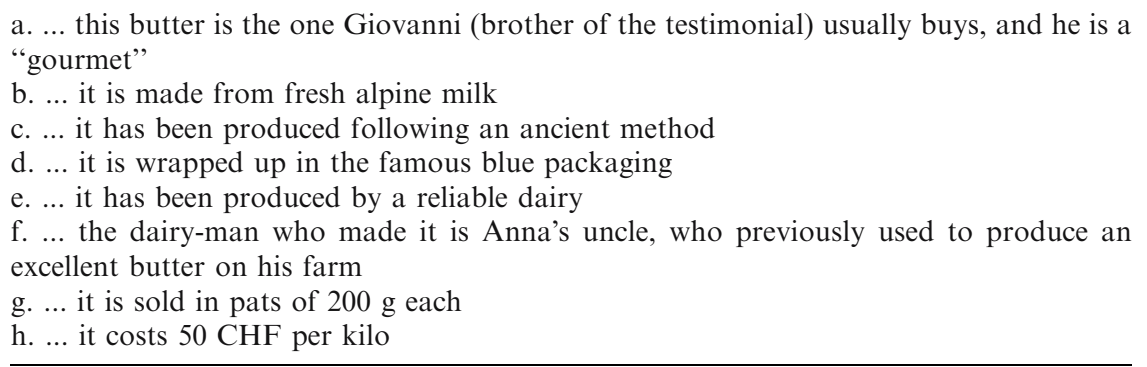


The heuristic use of the generator of arguments can have two different functions, with different relevance depending on the context and on the communicative habits. We will consider two particular situations that can well represent these two functions.

(1) In the first place, the generator works as a device that can reveal the argumentative power of the available information. This is done by taking into consideration the information items contained in the information protocol and by establishing their hooking point to the standpoint. In other words, the generator is used to define for each item of information in the information protocol whether it is relevant to the standpoint and the reason for its relevance, that is the nature of its hooking to the standpoint. If we want to build an argumentative move for the butter advertisement, we find in our information protocol two pieces of information - (d) and (g) - that are apparently irrelevant with regard to the standpoint. ${ }^{13}$ All the other pieces of information are instead possible arguments, because all of them can be included in a precise locus, thanks to their hooking to the standpoint: (b) can be included in the locus from the material cause, (c) in the locus from the instruments, (e) in the locus from the efficient cause, (f) similarly in the locus from the efficient cause, though in a more articulated way, (h) in the locus from implications (the price is implied by the value).

At this point it is a matter of establishing the actual argumentative power of each one of these possible arguments. This can only be done by means of the maxim, that we will reformulate here in order to use it as a heuristic device. The maxim we are going to use and that refers to the locus from the material cause (suggested, in the example, by information $b$ ), is the following:

"The quality of the product strongly depends on the quality of its material cause"

In its heuristic function the maxim becomes an instruction, which can be divided into three parts:

- Identify an endoxon that can show the positive quality of the material cause.

- Starting from this endoxon, build an enthymematic argumentation that shows that the quality of the material cause is positive.

- Use the conclusion of the enthymeme as minor premise for the maxim, thus producing the intended argumentative effect.

In other words, the maxim becomes a heuristic task: it leads to finding an endoxon that will guarantee argumentative power to the selected information. In the case of the locus from the material cause, the relevant endoxon is the one that states the fact that fresh alpine milk is a high quality material. 
It is clear that in cases similar to ours, where it is possible to have more than one relevant piece of information, and where each piece of information, if adequately included in an argumentative locus, can generate more than one argumentation in support of the standpoint, the argumentative strategy must include also a phase of evaluation of the arguments. This is not only a matter of evaluation of the logical force and efficacy of each single argument, but also of a compared evaluation of their relative efficacy, when it is necessary to select from a certain number of arguments, as well as of including them within a complex argumentative act (dispositio).

(2) The second function of the generator is to guide the search for information necessary to support the standpoint. Imagine, for example, a detective who, in order to establish whether a suspect had a possible motive for committing the homicide, must know whether the suspect received economic advantage from the death of the victim. Quite like the detective, a media analyst could consider the advantage of checking the reliability of a source, when evaluating a piece of news. Similarly, a bank, before providing a loan, must verify the client's capability of sustaining the debt. In this case it could be appropriate to inquire into the client's preceding behaviour towards banks, or into the reliability of the securities he is putting up for the loan (by checking, for example, that the properties he is offering as security do not derive from manipulative leverage activities).

\section{NOTES}

1 See Feteris (1987, 1993), who focuses on the role of the judge in the legal process.

2 This "faith" is reflected in the so-called ten rules of critical discussion, in particular in the "freedom rule" (van Eemeren and Grootendorst, 1992, pp. 208-209).

3 Argumentum is an online course on argumentation theory financed by the Swiss Virtual Campus (see www.argumentum.ch), and realized by the university of Lugano (responsible for the leading house, Eddo Rigotti, and project coordinator, Sara Greco Morasso), the University of Neuchâtel (Anne-Nelly Perret-Clermont), and the University of Geneva (Franz Schultheis), with the collaboration of the eLab Competence centre (Stefano Tardini) for the implementation of the online e-learning course.

4 For more details about modern contributions related to topics, in particular to the notion of argument scheme, see Rigotti and Greco (2006) and Rigotti (2007).

5 Agency theory, a key instrument in explaining many economic and social phenomena, was defined by Ross (1973) as follows: "We will say that an agency relationship has arisen between two (or more) parties when one, designated as the agent, acts for, on behalf of, or as representative for the other, designated as the principal, in a particular domain of decision problems". The principal delegates a task, which entails a decision making activity, to the agent, and the agent gets a compensation for it. In such kinds of relationship a problem arises (the agency problem) because there is no alignment of goals between the two parties, and the agent tends to act, as much as possible, in her own interest. Examples of agency relationship can be found in many situations and in different contexts of interaction (see Eisenhardt, 1989; Mann, 1997). 
${ }^{6}$ An introduction to logic tailored for students in argumentation theory, worked out by Marco Colombetti, is now being published within Argumentum.

7 The claim of generating all relevant arguments in relation to a certain standpoint might appear unreasonable. I am not pretending that our model of topics is able to actually produce all relevant arguments. As a matter of fact, no model of topics could ever be considered exhaustive in this sense, given that each fragment of reality shows endless aspects that bear endless relations with endless other fragments of reality... Nonetheless the system of topics generates all relevant arguments as it is expected to assign to each possible argument a precise inferential structure that is related to the ontology of the standpoint.

${ }^{8}$ It is often the case that the term argument is used to indicate both an argument and a locus. Thus, instead of saying "argument belonging to the locus from authority", one can speak of "argument from authority".

9 A certain likeliness with the locus from definition is evident, but an essential difference should not be neglected: the locus from definition necessarily refers to constitutive traits of the concerned entity, while, very often, the locus from derivates refers to aspects of the concerned entity whose relevance depends on the extent to which their scope covers the whole concerned entity. In our example, we notice that a professional status like being an entrepreneur does not exhaust (is only a part of) the civil status (with related rights and duties) of a citizen. When resting on a proper locus from definition, the arguments of this locus acquire indeed a quite different inferential strength: As they are human beings, they are expected to behave humanly. Evidently, the specific force of this locus is bound to the wording (is a sort of poetic proof) and is therefore rhetorical in nature, while its inferential strength depends each time on the locus that is exploited.

${ }^{10}$ Giuseppe Buffi (1938-2000) was member of the State Council of the Republic and Canton of Ticino and Director of the Department of education and culture from 1987 to 2000.

11 Terminus medius (middle term) was named, in the traditional logic, the term occurring in both premises of the syllogistic structure, that allows the connection between the subject and the predicate in the conclusion, but which must be absent from the conclusion.

12 Not considering other metaphorical interpretations that, in any case, should be specified, it is not possible to say, ${ }^{*}$ This dog is natural, or ${ }^{*}$ In this shop are sold natural clothes.

13 Of course, we can not exclude any possibility of argumentative exploitation of these information items; let us image that the colour of the package excites in the audience an emotion bound to a particular cultural value or that ...

\section{REFERENCES}

Aristotelis.: 1959, 'Ars Rhetorica', W. D. Ross (ed.), Oxford University Press, Oxford.

Aristotelis.: 1949, 'Categoriae et Liber de Interpretatione', L. Minio-Paluello (ed.), Oxford University Press, Oxford.

Aristotelis.: 1958, 'Topica et Sophistici Elenchi', W. D. Ross (ed.), Oxford University Press, Oxford.

Cantoni, L. and N. Di Blas: 2006, (2nd edition), Comunicazione. Teoria e Pratiche, Apogeo, Milano.

van Eemeren, F. H. and R. Grootendorst: 1992, Argumentation, Communication, and Fallacies, Lawrence Erlbaum Associates, Hillsdale, New Jersey.

van Eemeren, F. H. and R. Grootendorst: 2004, A Systematic Theory of Argumentation: The Pragma-dialectical Approach, Cambridge University Press, Cambridge.

van Eemeren, F. H. and P. Houtlosser: 2005b, 'Theoretical Construction and Argumentative Reality: An Analytic Model of Critical Discussion and Conventionalised Types of Argumentative Activity', in D. Hitchcock and D. Farr (eds.), The Uses of Argument. Proceedings of a Conference at McMaster University, 18-21 May 2005, pp. $75-84$. 
van Eemeren, F. H. and P. Houtlosser: 2002, 'Strategic Maneuvering: Maintaining a Delicate Balance', in F. H. van Eemeren and P. Houtlosser (eds.), Dialectic and Rhetoric. The Warp and Woof of Argumentation Analysis, Kluwer Academic Publisher, Amsterdam.

van Eemeren F. H. and P. Houtlosser: 2005a, 'Strategic Manoeuvring', in M. Dascal, F. H. van Eemeren, E. Rigotti, S. Stati and A. Rocci (eds.), Argumentation in Dialogic Interaction, Studies in Communication Sciences, pp. 1-20, special issue, Lugano.

Eisenhardt, K. M.: 1989, 'Agency Theory: An Assessment and Review', The Academy of Management Review 14, 57-74.

Feteris, E. T.: 1987, 'The Dialectical Role of the Judge in a Legal Process', in J. W. Wenzer (ed.), Argument and Critical Practices. Proceedings of the Fifth SCA/AFA Conference on Argumentation, Speech Communication Association, Annandale.

Feteris, E. T.: 1993, 'The Judge as a Critical Antagonist in a Legal Process: A Pragmadialectical Perspective', in R. E. McKenow (ed.), Argument and the Postmodern Challenge. Proceedings of the Eight SCA/AFA Conference on Argumentation, Speech Communication Association, Annandale.

Greco Morasso, S.: forthcoming, Argumentative and Other Communicative Strategies of the Mediation Practice, PhD thesis, University of Lugano.

Greco Morasso, S.: 2007 (in press), 'Towards a Multidisciplinary Context-Dependent Model of Mediation Practice', in M. Colombetti (ed.), Communication Sciences as a Multidisciplinary Enterprise. Special Issue of Studies in Communication Sciences.

Hispani, P.: 1947, 'Summulae Logicales', M. Bochensky (ed.), Marietti, Torino.

Hubbel, H. M.: 1949, Cicero: On inventio - On the Best Kind of Orator - Topics, Harvard University Press, Cambridge, MA.

Levinson, S. C.: 1978, 'Activity Types and Language', Pragmatics Microfiche Volume 3, Fiche 3-3, D.1-G.5. Reprinted in Levinson, S.C.: 1979, 'Activity Types and Language', Linguistics 17, 365-399.

Mann, S.: 1997, 'Agency theory', in J. Garrett (ed.), The Blackwell Enclyclopedia of Management, Second Edition, Blackwell Publishing, Malden.

Petty, R. E. and J. T. Cacioppo: 1986, Communication and Persuasion: Central and Peripheral Routes to Attitude Change, Springer, New York.

Petty, R. E., J. T. Cacioppo and D. T. Schumann: 1983, 'Central and Peripheral Routes to Advertising Effectiveness: The Moderating Effect of involvement', Journal of Consumer Research 10, 135-146.

Quintiliani, M. F.: 1970, Institutions oratoriae libri duodecim, M. Winterbottom (ed.), Oxford University Press, Oxford.

Rigotti, E. and A. Rocci: 2001, 'Sens - Non-sens - Contresens. Tentative d'une Définition Explicative', Studies in Communication Sciences 1, 45-80.

Rigotti, E. and A. Rocci.: 2007 (in press), 'Towards a Definition of Communication Context', in M. Colombetti (ed.), Communication Sciences as a Multidisciplinary Enterprise, Special Issue of Studies in Communication Science.

Rigotti, E. and S. Greco: 2006, Topics: The Argument Generator, Argumentum eLearning Module, www.argumentum.ch

Rigotti, E.: 2007 (in press), 'Can Classical Topics be Revived Within the Contemporary Theory of Argumentation?', in F. H. van Eemeren, A. J. Blair, F. Snoeck Henkemans and Ch. Willards (eds.), Proceedings of the Sixth Conference of the International Society for the Study of Argumentation, Sic Sat, Amsterdam.

Ross, S. A.: 1973, 'The Economic Theory of Agency: The Principal's Problem', The American Economic Review, 63(2), Papers and Proceedings of the Eighty-fifth Annual Meeting of the American Economic Association, May 1973, pp. 134-139.

Searle, J. R.: 1996, The construction of social reality, Penguin, London.

Stump, E.: 2004, Boethius's 'De Topicis Differentiis', Cornell University Press, Ithaca, NY. 